\title{
ТЕОРИЯ ДВУХСТУПЕНЧАТОГО ИМПУЛЬСНОГО ФОТОВЫЖИГАНИЯ ПРЕДЕЛЬНО УЗКИХ СПЕКТРАЛЬНЫХ ПРОВАЛОВ
}

(Представил В. Хижняков)

\section{1. Введение}

Фотовыжигание узких провалов шириной до $10^{-3} \mathrm{~cm}^{-1}$ в спектрах примесных систем при стационарном возбуждении в неоднородной полосе чисто электронного перехода примесного центра широко используется как метод устранения неоднородного уширения спектров $\left[{ }^{1-4}\right]$. Фотовыжигание спектральных провалов (ФСП) импульсами пикосекундной длительности позволяет осуществлять параллельное выжигание многих спектральных провалов (СП) и лежит в основе спектрально-временной голографической записи информации в неоднородно уширенной полосе поглощения двухуровневой системы $\left[{ }^{5}\right]$. Оптическая информация, записанная путем одноступенчатого фотовыжигания, неизбежно портится при считывании. Это можно избежать, если использовать трехуровневые системы с двухступенчатым выжиганием, где селективное возбуждение первой ступени «закрепляется» фотохимическим превращением через вторую ступень возбуждения $\left[{ }^{6-8}\right]$. В данной работе двухступенчатое ФСП импульсами рассмотрено с позиций теории переходных (зависящих от времени) спектров вторичного свечения $\left[{ }^{9}\right]$. Теоретически показана возможность дальнейшего сужения СП в трехуровневых системах при введении временной задержки $T$ между селектирующим и закрепляющим импульсами.

\section{2. Провал в функции неоднородного распределения}

Кинетику образования провала удобно рассматривать как преобразование функции неоднородного распределения (ФНР) центров по частоте данного перехода $\varrho(\Omega, t)\left[{ }^{3}\right]$. В рассматриваемом случае трехуровневых систем необходимо ввести двухмерную ФНР, учитывающую неоднородное распределение частот $\Omega_{01}$ и $\Omega_{12}$ переходов $0 \rightarrow 1$ и $1 \rightarrow 2$. Пусть функция $\varrho_{0}\left(\Omega_{01}, \Omega_{12}\right)$ описывает двухмерное неоднородное распределение, существовавшее до взаимодействия центров со световыми импульсами, причем области частот $\Omega_{01}$ и $\Omega_{12}$ не перекрываются. Пусть на систему падают последовательно два импульса, из которых первый переводит систему с уровня 0 на уровень 1, а второй переводит систему с уровня 1 на уровень 2 , где с вероятностью $\alpha$ происходит фотопревращение центра, выводящее его из резонанса с обоими импульсами.

Возникающая в результате этого двухмерная ФНР изменяется во времени по экспоненциальному закону, если выполняются некоторые условия $\left[{ }^{3}\right]$ : 
где

$$
P\left(\Omega_{01}, \Omega_{12}, t\right)=\alpha \int_{-\infty}^{t} \mathrm{dt}^{\prime} W\left(\Omega_{01}, \Omega_{12}, t^{\prime}\right) .
$$

Здесь $W\left(\Omega_{01}, \Omega_{12}, t^{\prime}\right)$ - вероятность нахождения молекулы на уровне 2 в момешт времени $t^{\prime}$, а $P\left(\Omega_{01}, \Omega_{12}, t\right)$ - вероятность выхода центра из резонанса с возбуждающими импульсами к моменту $t$ (вероятность ФСП). При условии достаточно малой интенсивности возбуждения можно обеспечить выполнимость условия $W\left(\Omega_{01}, \Omega_{12}, t^{\prime}\right) \ll 1$ и рассмот. реть процесс двухступенчатого возбуждения во втором порядке теории возмущений, учитывая только один акт последовательного поглощения двух импульсов $\left[{ }^{9}\right]$ :

$$
\begin{gathered}
W\left(\Omega_{01}, \Omega_{12}, t^{\prime}\right)=\iint_{-\infty}^{t^{\prime}} \mathrm{dt}_{1} \mathrm{dt}_{1}^{\prime} S_{2}\left(t_{1}, t_{1}^{\prime}\right) \int_{-\infty}^{t_{1}} \mathrm{dt}_{2} \int_{-\infty}^{t_{1}^{\prime}} \mathrm{dt}_{2}^{\prime} S_{1}\left(t_{2}, t_{2}^{\prime}\right) \times \\
\times F\left(t_{1}^{\prime}-t_{1}, t^{\prime}-t_{1}^{\prime}, t_{1}^{\prime}-t_{2}^{\prime}, t_{1}-t_{2}\right),
\end{gathered}
$$

где $S_{1}\left(t_{2}, t_{2}{ }^{\prime}\right)$ и $S_{2}\left(t_{1}, t_{1}{ }^{\prime}\right)$ - корреляционные функции первого и второго (селектирующего и закрепляющего) светового импульса,

$$
\begin{gathered}
F\left(t_{1}^{\prime}-t_{1}, t^{\prime}-t_{1}^{\prime}, t_{1}^{\prime}-t_{2}^{\prime}, t_{1}-t_{2}\right)=\left\langle v_{\omega_{1}}^{+} \exp \left[i\left(\hat{H}+\frac{i}{2} \hat{\gamma}\right)\left(t_{1}-t_{2}\right)\right] \times\right. \\
\quad \times v_{\omega_{2}}^{+} \exp \left[i \hat{H}\left(t_{1}^{\prime}-t_{1}\right)-\frac{1}{2} \hat{\gamma}\left(2 t^{\prime}-t_{1}-t_{1}^{\prime}\right)\right] v_{\omega_{2}} \times \\
\left.\quad \times \exp \left[-i\left(\hat{H}-\frac{i}{2} \hat{\gamma}\right)\left(t_{1}^{\prime}-t_{2}^{\prime}\right)\right] v_{\omega_{1}} \exp \left[-i \hat{H}\left(t_{2}^{\prime}-t_{2}\right)\right]\right\rangle
\end{gathered}
$$

- корреляционная функция примесного центра (трехуровневой системы). Здесь

и

$$
v_{\omega_{1}}=\left\langle N-1_{\omega_{1}}|\hat{V}| N\right\rangle
$$

$$
v_{\omega_{2}}=\left\langle N-1_{\omega_{1}}-1_{\omega_{2}}|\hat{V}| N-1_{\omega_{1}}\right\rangle
$$

однофотонные матричные элементы гамильтониана взаимодействия $\hat{V}$ поля и вещества, описывающие уничтожение фотонов частоты $\omega_{1}$ и $\omega_{2}$ $\left(\omega_{1}-\right.$ средняя частота первого возбуждающего импульса, $\omega_{2}-$ средняя частота второго импульса (условия резонанса $\omega_{1} \approx \Omega_{01}$ и $\omega_{2} \approx \Omega_{12}$ ), $|N\rangle$ - исходное состояние электромагнитного поля, $\hat{H}-$ гамильтониан вещества (примесного центра), $\hat{\gamma}$ - оператор радиационного затухания трехуровневой системы; $\langle\ldots\rangle-$ - знак усреднения по ансамблю колебаний на начальном электронном уровне.

Вероятность $W\left(\Omega_{01}, \Omega_{12}, t^{\prime}\right)$ можно записать также в виде $W\left(\Omega_{01}, \Omega_{12}, t^{\prime}\right)=\iiint \int d \omega_{1} d \omega_{2} d \omega_{1}^{\prime} d \omega_{2}^{\prime} \widetilde{S}_{1}\left(\omega_{1}, \omega_{1}^{\prime}\right) \widetilde{S}_{2}\left(\omega_{2}, \omega_{2}^{\prime}\right) \times$

$$
\begin{aligned}
& \times \iint_{-\infty}^{t^{\prime}} \mathrm{dt}_{1} \mathrm{dt}_{1}^{\prime} \int_{-\infty}^{t_{1}} \mathrm{dt}_{2} \int_{-\infty}^{t_{1}^{\prime}} \mathrm{dt}_{2}^{\prime} F\left(t_{1}^{\prime}-t_{1}, t^{\prime}-t_{1}^{\prime}, t_{1}^{\prime}-t_{2}^{\prime}, t_{1}-t_{2}\right) \times \\
& \times \exp \left(i \omega_{1} t_{2}-i \omega_{1}^{\prime} t_{2}^{\prime}+i \omega_{2} t_{1}-i \omega_{2}^{\prime} t_{1}^{\prime}\right),
\end{aligned}
$$

где функции $\widetilde{S}_{1}\left(\omega_{1}, \omega_{1}^{\prime}\right)$ и $\widetilde{S}_{2}\left(\omega_{2}, \omega_{2}^{\prime}\right)$ введены соотношениями

и

$$
\begin{aligned}
& S_{1}\left(t_{2}, t_{2}^{\prime}\right)=\iint d \omega_{1} d \omega_{1}^{\prime} S_{1}\left(\omega_{1}, \omega_{1}^{\prime}\right) \exp \left(i \omega_{1} t_{2}-i \omega_{1}^{\prime} t_{2}^{\prime}\right) \\
& S_{2}\left(t_{1}, t_{1}^{\prime}\right)=\iint d \omega_{2} d \omega_{2}^{\prime} S_{2}\left(\omega_{2}, \omega_{2}^{\prime}\right) \exp \left(i \omega_{2} t_{1}-i \omega_{2}^{\prime} t_{1}^{\prime}\right) .
\end{aligned}
$$


Используем в (5) новые переменные $\mu=t_{i}^{\prime}-t_{1}, v=t^{\prime}-\frac{1}{2}\left(t_{1}+t_{1}^{\prime}+|\mu|\right)$, $\tau=t_{1}^{\prime}-t_{2}^{\prime}, \tau^{\prime}=t_{1}-t_{2}$. Получим вероятность ФСП $\left[{ }^{10,11}\right]$

$$
P\left(\Omega_{01}, \Omega_{12}, t\right)=2 \alpha \operatorname{Re}\left\{\int_{-\infty}^{t} \mathrm{dt}^{\prime} \iiint \int d \omega_{1} d \omega_{2} d \omega_{1}^{\prime} d \omega_{2}^{\prime} \widetilde{S}_{1}\left(\omega_{1}, \omega_{1}^{\prime}\right) \times\right.
$$

$\times \widetilde{S}_{2}\left(\omega_{2}, \omega_{2}^{\prime}\right) \iiint_{0}^{\infty} \int d \mu d \nu d \tau d \tau^{\prime} F\left(\mu, v, \tau, \tau^{\prime}\right) \exp \left[i \omega_{1}\left(t^{\prime}-\mu-v-\tau^{\prime}\right)-\right.$

$$
\left.\left.-i \omega_{1}^{\prime}\left(t^{\prime}-v-\tau\right)+i \omega_{2}\left(t^{\prime}-\mu-v\right)-i \omega_{2}^{\prime}\left(t_{1}-v\right)\right]\right\} .
$$

Переходя к пределу $t \rightarrow \infty \quad\left(P\left(\Omega_{01}, \Omega_{12}\right) \equiv \lim P\left(\Omega_{01}, \Omega_{12}, t\right)\right)$, получим $P\left(\Omega_{01}, \Omega_{12}\right)=2 \alpha \operatorname{Re}\left\{\iiint d \omega_{1} d \omega_{2} d \omega_{1}^{\prime} \widetilde{S}_{1}\left(\omega_{1}, \omega_{1}^{\prime}\right) \widetilde{S}_{2}\left(\omega_{2}, \omega_{1}+\omega_{2}-\omega_{1}^{\prime}\right) \times\right.$

$\left.\times \iiint \int d \mu d \nu d \tau d \tau^{\prime} F\left(\mu, v, \tau, \tau^{\prime}\right) \exp \left[-i \omega_{1}\left(\mu+\tau^{\prime}\right)+i \omega_{1}^{\prime} \tau-i \omega_{2} \mu\right]\right\}$.

Вероятность выжигания $P\left(\Omega_{01}, \Omega_{12}\right)$ с помощью формулы (1) определяет окончательный провал в $Ф Н \mathrm{Q} \varrho\left(\Omega_{01}, \Omega_{12}\right)$ при выжигании двумя световыми импульсами, имеющими среднюю частоту в области $\Omega_{01}$ и $\Omega_{12}$, соответственно.

В случае стационарного ФСП светом с произвольным спектральным распределением в промежутке времени $(0, t)\left(t \gg \gamma^{-1}\right)$ вероятность выжигания

$$
P_{\text {ст }}\left(\Omega_{01}, \Omega_{12}, t\right)=\alpha t \iint d \omega_{1} d \omega_{2} \widetilde{S}_{1}\left(\omega_{1}\right) \widetilde{S}_{2}\left(\omega_{2}\right) \times
$$

$\times 2 \operatorname{Re}\left\{\iiint_{0}^{\infty} \int d \mu d v d \tau d \tau^{\prime} F\left(\mu, v, \tau, \tau^{\prime}\right) \exp \left[i \omega_{1}\left(\tau-\tau^{\prime}-\mu\right)-i \omega_{2} \mu\right]\right\}$,

где $\widehat{S}(\omega)$ - интенсивность возбуждающего света на частоте $\omega$. Таким образом, сравнивая формулы (8) и (9), видим, что при двухступенчатом импульсном ФСП вероятности выжигания, а отсюда и получаемые спектральные провалы существенно различны при выжигании импульсами и при стационарном выжигании. Рассмотрение одноступенчатого импульсного ФСП показывает, что при условии совпадения распределения частот и суммарной дозы облучения выжигание одиночным импульсом и выжигание в стационарном режиме приводит к образованию одинаковых провалов (см. Приложение).

В заключение рассмотрим случай, где первый этап выжигания $0 \rightarrow 1$ происходит импульсом, а второй $1 \rightarrow 2-$ стационарным светом. Вероятность ФСП следующая:

$$
P\left(\Omega_{01}, \Omega_{12}\right)=\alpha \int d \omega_{2} \widetilde{S}_{2}\left(\omega_{2}\right) \int d \omega_{1} \widetilde{S}_{1}\left(\omega_{1}, \omega_{1}\right) \times
$$

$$
\times 2 \operatorname{Re}\left\{\iint_{0}^{\infty} \iint d \mu d \nu d \tau d \tau^{\prime} F\left(\mu, v, \tau, \tau^{\prime}\right) \exp \left[i \omega_{1}\left(\tau-\tau^{\prime}-\mu\right)-i \omega_{2} \mu\right]\right\} .
$$

Сравнивая формулы (9) и (10) видим, что если равенство $t \tilde{S}_{1}\left(\omega_{1}\right)=$ $=\widetilde{S}_{1}\left(\omega_{1}, \omega_{1}\right)$ выполняется при каждой частоте $\omega_{1}$, то в рассматриваемом случае образуется такой же провал, как и в случае стационарного выжигания.

\section{3. Случай короткого закрепляющего импульса}

Пусть при ФСП закрепляющий импульс существенно короче селектирующего импульса, времен релаксации уровней 1 и 2, а также спект- 
рально шире неоднородного распределения частоты $\Omega_{12}$. В этом случае можно положить

$$
S_{2}\left(t_{1}, t_{1}^{\prime}\right)=S_{20} \delta\left(t_{1}-\tau_{2}\right) \delta\left(t_{1}^{\prime}-\tau_{2}\right),
$$

где $S_{20}$ - константа, $\tau_{2}-$ момент прохождения центра закрепляющим импульсом. Из формул (2) и (3) получаем $(t \rightarrow \infty)$ вероятность выжигания $P\left(\Omega_{10}\right)$, определяющую провал в $Ф \mathrm{HP} Q\left(\Omega_{01}\right)$ :

$P\left(\Omega_{01}\right)=\alpha S_{20} \int_{\tau_{2}}^{\infty} \mathrm{dt}^{\prime} \int_{-\infty}^{\tau_{2}} \int_{-\infty} \mathrm{dt}_{2} \mathrm{dt}_{2}^{\prime} S_{1}\left(t_{2}, t_{2}^{\prime}\right) F\left(0, t^{\prime}-\tau_{2}, \tau_{2}-t_{2}^{\prime}, \tau_{2}-t_{2}\right)$.

Предположим, что корреляционная функция селектирующего импульса следующая (импульс когерентный и затухает по экспоненциальному закону):

$$
\begin{gathered}
S_{1}\left(t_{2}, t_{2}^{\prime}\right)=\theta\left(t_{2}-\tau_{1}\right) \theta\left(t_{2}^{\prime}-\tau_{1}\right) \Delta \exp \left[i \omega_{0}\left(t_{2}-t_{2}^{\prime}\right)-\right. \\
\left.-\frac{\Delta}{2}\left(t_{2}+t_{2}^{\prime}-2 \tau_{1}\right)\right],
\end{gathered}
$$

$\left(\tau_{1}<\tau_{2}\right)$, где $\tau_{1}-$ начальный момент прохождения центра селектирующего импульса, $\omega_{0}$ и $\Delta-$ его частота максимума и спектральная ширина, т. е. полная ширина на половине высоты.

Для описания трехуровневой системы (примесного центра) используем модель, в которой релаксационные процессы на возбужденном уровне 1 описываются константами энергетической (продольной) и фазовой (поперечной) релаксации $\gamma_{1}$ и $\Gamma$, на уровне $2-$ константой энергетической релаксации $\gamma_{2}$. (В рассматриваемом случае учет модуляционного уширения (фазовой релаксации) уровня 2 не существен.) Соответствующая корреляционная функция трехуровневой системы следующая:

$$
\begin{gathered}
F\left(t_{1}^{\prime}-t_{1}, t^{\prime}-t_{1}, t_{1}^{\prime}-t_{2}^{\prime}, t_{1}-t_{2}\right)=C \exp \left[-\gamma_{2} t^{\prime}-i \Omega_{12}\left(t_{1}-t_{1}^{\prime}\right)+\right. \\
+\frac{\gamma_{2}}{2}\left(t_{1}+t_{1}^{\prime}\right)-i \Omega_{01}\left(t_{2}-t_{2}^{\prime}\right)-\frac{\gamma_{1}}{2}\left(t_{1}+t_{1}^{\prime}-t_{2}-t_{2}^{\prime}\right)- \\
\left.-\frac{\Gamma}{2}\left(t_{1}-t_{2}+t_{1}^{\prime}-t_{2}^{\prime}+\left|t_{1}-t_{1}^{\prime}\right|+\left|t_{2}-t_{2}^{\prime}\right|-\left|t_{1}-t_{2}^{\prime}\right|-\left|t_{1}^{\prime}-t_{2}\right|\right)\right] .
\end{gathered}
$$

( $C$ - константа. В модели фононные крылья не учитываются.)

При малых дозах облучения и после фотовыжигания ФНР $\varrho\left(\Omega_{01}\right)$ будет иметь вид $\left[{ }^{11}\right]$ :

$$
\mathrm{Q}\left(\Omega_{01}\right) \approx \mathrm{Q}_{0}\left(\Omega_{01}\right)\left[1-P\left(\Omega_{01}\right)\right],
$$

где

$$
\begin{gathered}
P\left(\Omega_{01}\right)=P_{2}\left(\Omega_{01}\right)=\varepsilon \Delta\left\{\exp (-\Delta T) \frac{\Gamma+a}{a \xi_{1}}-\exp \left(-\gamma_{1} T\right) \frac{\Gamma-a}{a \xi_{2}}-\right. \\
-\exp \left[-\frac{1}{2}\left(\Gamma+\gamma_{1}+\Delta\right) T\right] \frac{2}{\xi_{1} \xi_{2}}\left[\left(x^{2}-\frac{1}{4}\left(\Gamma^{2}-a^{2}\right)\right) \cos (x T)+\right. \\
+\Gamma x \sin (x T)]\},
\end{gathered}
$$

где $\varepsilon=\alpha S_{20} C \gamma_{2}^{-1}, \quad T=\tau_{2}-\tau_{1}, x=\Omega_{01}-\omega_{0}, a=\gamma_{1}-\Delta, \xi_{1}=x^{2}+\frac{1}{4}(\Gamma+a)^{2}$ и $\xi^{2}=x^{2}+\frac{1}{4}(\Gamma-a)^{2}$. Из формулы (16) видим, что провал в ФНР 
монотонно сужается с ростом времени задержки $T$ между выжигающими импульсами до предельной ширины $\sigma_{2}=\Gamma+\left|\gamma_{1}-\Delta\right|$ (см. рис. 1). Отметим, что в двухуровневой системе ширина СП в этой модели при выжигании импульсом $\sigma_{1}=\Gamma+\gamma_{1}+\Delta$ и при выжигании монохроматическим светом $\sigma_{0}=\Gamma+\gamma_{1}$. Если $T^{-1}$ и $\left|\gamma_{1}-\Delta\right| \ll \gamma_{1}$ и $\Delta$, то в трехуровневой системе ширина СП меньше как $\sigma_{1}$, так и $\sigma_{0}$. Если также $\Gamma<\gamma_{1}$ и $\Delta$, то рассматриваемый провал может быть у́же и $\gamma_{1}$ и $\Delta$. Таким образом, имеет место эффект компенсации спектральной ширины селектирующего импульса и ширины, обусловленной затуханием возбужденного уровня 1. Этот эффект аналогичен эффекту компенсации в зависящем от времени спектре резонансного вторичного свечения [ $\left.{ }^{9}\right]$. Формула (16) аналогична формуле, описывающей зависящий от времени спектр люминесценции (или поглощения) $\left[{ }^{9}\right]$. Модуляционное уширение уровня $1-\Gamma$ не компенсируется, а прибавляется $\mathrm{K}$ величине $\left|\gamma_{1}-\Delta\right|$.

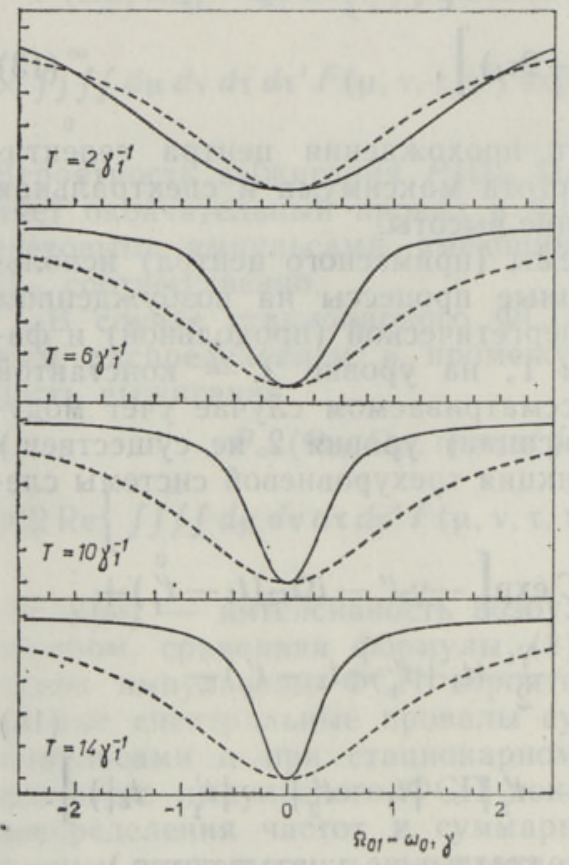

Рнс. 1. Провал в ФНР $\mathrm{Q}\left(\Omega_{01}\right)$ при двухступенчатом (сплошная линия) и одноступенчатом (штрнховая линия) ФСП. Параметры: $\Gamma=0,5 \gamma_{1}, \quad \Delta=0,99 \gamma_{1} \quad\left(\sigma_{0}=\right.$ $\left.=1,5 \gamma_{1}, \quad \sigma_{1}=2,49 \gamma_{1}, \sigma_{2}=0,51 \gamma_{1}\right)$.

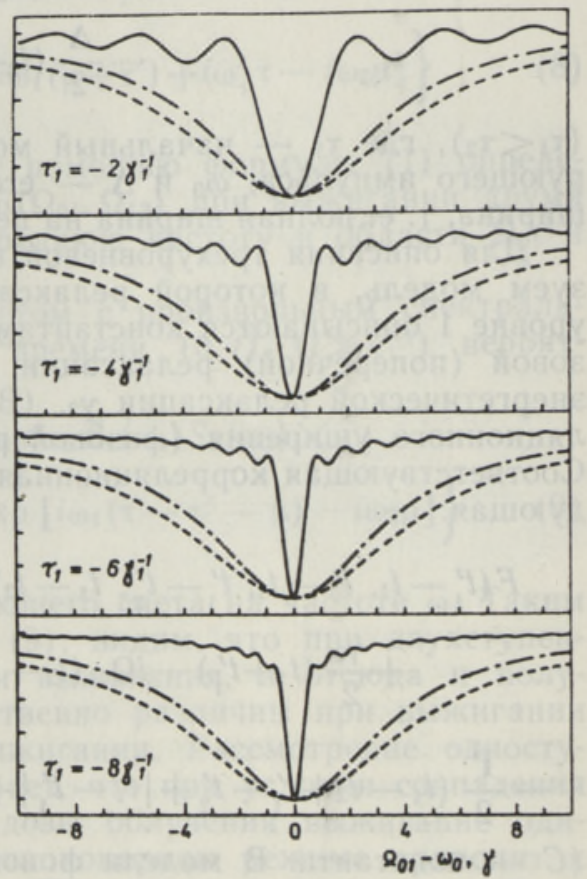

Рис. 2. Провал в ФНР $\mathrm{e}\left(\Omega_{01}\right)$ при одноступенчатом (штриховая линия) и двухступенчатом (штрих-пунктир) ФСП селектирующим импульсом, а также при двухступенчатом ФСП суммой селектирующего импульса и добавочного $\delta$-импульса (сплошная линия). Параметры: $\Gamma=4 \gamma_{1}$, $\Delta=4,5 \gamma_{1}, \quad \tau_{2}=\gamma_{1}^{-1} \quad\left(\sigma_{0}=5 \gamma_{1}, \quad \sigma_{1}=9,5 \gamma_{1}\right.$, $\left.\sigma_{2}=7,5 \gamma_{1}, \sigma_{3}=0,5 \gamma_{1}\right)$.

\section{4. Использование интерференции для дальнейшего сужения провала}

На первой ступени; выжигания добавим к селектирующему импульсу (см. (13)) предельно короткий импульс (ס-импульс), проходящий центр в момент времени нуль. (Точнее добавочный импульс должен быть существенно короче селектирующего импульса, времен релаксации уровня 1, а также спектрально шире неоднородного распределения частоты $\Omega_{01}$.) В этом случае корреляционная функция, описывающая выжигающее световое поле̨ на первой ступени выжигания, имеет вид 


$$
S_{1}\left(t_{2}, t_{2}^{\prime}\right)=\delta\left(t_{2}\right) \delta\left(t_{2}^{\prime}\right) S_{10}+\theta\left(t_{2}-\tau_{1}\right) \theta\left(t_{2}^{\prime}-\tau_{1}\right) \Delta \times
$$

$$
\begin{gathered}
\times \exp \left[i \omega_{0}\left(t_{2}-t_{2}^{\prime}\right)-\frac{\Delta}{2}\left(t_{2}+t_{2}^{\prime}-2 \tau_{1}\right)\right]+\delta\left(t_{2}^{\prime}\right) \theta\left(t_{2}-\tau_{1}\right) \sqrt{S_{10} \Delta} \times \\
\times \exp \left[i \omega_{0} t_{2}-\frac{\Delta}{2}\left(t_{2}-\tau_{1}\right)\right]+\delta\left(t_{2}\right) \theta\left(t_{2}^{\prime}-\tau_{1}\right) \overline{S_{10} \Delta} \times \\
\times \exp \left[-i \omega_{0} t_{2}^{\prime}-\frac{\Delta}{2}\left(t_{2}^{\prime}-\tau_{1}\right)\right] .
\end{gathered}
$$

Подставляя формулы (17) и (14) в формулу (12) и интегрируя по формуле (12), получаем вероятность выжигания $P\left(\Omega_{01}\right)$ в виде следующих трех слагаемых $\left[{ }^{12}\right]$ :

$$
P\left(\Omega_{01}\right)=P_{1}+P_{2}\left(\Omega_{01}\right)+P_{3}\left(\Omega_{01}\right) .
$$

Здесь

$$
P_{1}=\varepsilon S_{10} \exp \left(-\gamma_{1} \tau_{2}\right)
$$

не зависит от частоты перехода $\Omega_{01}$ и соответствует выжиганию добавочным $\delta$-импульсом. Вероятность $P_{2}\left(\Omega_{01}\right)$ соответствует выжиганию селектирующим импульсом и определяется формулой (16). Вероятность $P_{3}\left(\Omega_{01}\right)$ соответствует выжиганию интерференционным членом, возникшим вследствие интерференции селектирующего и добавочного импульсов

$$
\begin{gathered}
P_{3}\left(\Omega_{01}\right)=\varepsilon \sqrt{S_{10} \Delta} \exp \left(-\gamma_{1} \tau_{2}+\frac{\Delta}{2} \tau_{1}\right)\left\{\left[(a+\Gamma)-\exp \left(\frac{a+\Gamma}{2} \tau_{1}\right) \times\right.\right. \\
\left.\times\left((a+\Gamma) \cos \left(x \tau_{1}\right)+2 x \sin \left(x \tau_{1}\right)\right)\right] \xi_{1}^{-1}+\left[\exp \left(\frac{a-\Gamma}{2} \tau_{2}\right) \times\right. \\
\left.\left.\times\left((a-\Gamma) \cos \left(x \tau_{2}\right)+2 x \sin \left(x \tau_{2}\right)\right)-(a-\Gamma)\right] \xi_{2}^{-1}\right\}, \text { при } \tau_{1}<0 \\
P_{3}\left(\Omega_{01}\right)=\varepsilon \sqrt{S_{10} \Delta \exp }\left(-\gamma_{1} \tau_{2}+\frac{\Delta}{2} \tau_{1}\right)\left\{\exp \left(\frac{a-\Gamma}{2} \tau_{2}\right) \times\right. \\
\times\left[(a-\Gamma) \cos \left(x \tau_{2}\right)+2 x \sin \left(x \tau_{2}\right)\right]- \\
\left.-\exp \left(\frac{a-\Gamma}{2} \tau_{1}\right)\left[(a-\Gamma) \cos \left(x \tau_{1}\right)+2 x \sin \left(x \tau_{1}\right)\right]\right\} \xi_{2}^{-1}, \text { при } \tau_{1} \geqslant 0 .
\end{gathered}
$$

Из (20) следует, что в случае $\tau_{1}<0$ (см. рис. 2) провал, определяемый вероятностью выжигания $P_{3}\left(\Omega_{01}\right)$, сужается с ростом времени $\left|\tau_{1}\right|$ до предельной ширины $\sigma_{3}=\left|\gamma_{1}+\Gamma-\Delta\right|$. Таким образом, при каждых значениях $\gamma_{1}$ и $\Gamma$ можно подобрать $\Delta$ так, чтобы выполнялось условие $\gamma_{1}+\Gamma-\Delta=0$. Тогда с ростом времени $\left|\tau_{1}\right|$ ширина провала (точнее, центрального минимума) в ФНР, определяемого вероятностью $P_{3}\left(\Omega_{01}\right)$, стремится к нулю. Если $P_{3}\left(\Omega_{01}\right) \gg P_{2}\left(\Omega_{01}\right)$, то и ширина суммарного провала, определяемого суммой вероятностей $P_{2}\left(\Omega_{01}\right)+$ $+P_{3}\left(\Omega_{01}\right)$, с ростом $\left|\tau_{1}\right|$ стремится к нулю. Для выполнения условия $P_{3}\left(\Omega_{01}\right) \gg P_{2}\left(\Omega_{01}\right)$ с ростом $\left|\tau_{1}\right|$ необходимо уменьшить параметр $d \equiv \sqrt{\Delta / S_{10}}$.

В случае $\tau_{1} \geqslant 0$ провал, определяемый вероятностью выжигания $P_{3}\left(\Omega_{01}\right)$, сужается с ростом времени задержки $T=\tau_{2}-\tau_{1}$ до предельной ширины $\sigma_{4}=\left|\gamma_{1}-\Gamma-\Delta\right|$. При каждом $\Gamma<\gamma_{1}$ можно подобрать $\Delta$ таким образом, чтобы выполнялось условие $\gamma_{1}-\Gamma-\Delta=0$. Тогда с ростом времени $T$ ширина провала в $Ф Н Р$, определяемого вероятностью $P_{3}\left(\Omega_{01}\right)$, стремится к нулю. При $\gamma_{1} \leqslant \Gamma$ наиболее узкие провалы получаются при $\Delta=0$, что соответствует включению незатухающего возбуждения в момент времени $\tau_{1}$, 


\section{5. Увеличение спектрального разрешения метода выжигания провалов}

Рассмотрим выжигание провалов в ФНР $\varrho\left(\Omega_{01}\right)$ одновременно двумя одинаковыми селектирующими импульсами на частотах $\omega_{0}$ и $\omega_{0}+\omega_{1}$. Корреляционная функция, описывающая выжигающее световое поле, имеет вид:

$$
\begin{gathered}
S\left(t_{2}, t_{2}^{\prime}\right)=\theta\left(t_{2}-\tau_{1}\right) \theta\left(t_{2}^{\prime}-\tau_{1}\right) \Delta \exp \left[-\frac{\Delta}{2}\left(t_{2}+t_{2}^{\prime}-2 \tau_{1}\right)\right] \times \\
\times\left[\exp \left(i \omega_{0} t_{2}\right)+\exp \left(i\left(\omega_{0}+\omega_{1}\right) t_{2}\right)\right]\left[\exp \left(-i \omega_{0} t_{2}^{\prime}\right)+\exp \left(-i\left(\omega_{0}+\omega_{1}\right) t_{2}^{\prime}\right)\right] .
\end{gathered}
$$

Частотное распределение импульсов состоит из двух лоренцианов (с шириной $\Delta$ ) и интерференционного члена.

При ФСП в двухуровневой системе в рассматриваемом случае выжигаются либо два провала с максимумами на частотах $\Omega_{01}=\omega_{0}$ и $\Omega_{01}=\omega_{0}+\omega_{1}$ и ширинами $\sigma_{1}=\gamma_{1}+\Gamma+\Delta$, либо один провал с максимумом на частоте $\Omega_{01}=\omega_{0}+\omega_{1} / 2$ (два провала сливаются в один).

Подставляя формулы (14) и (21) в формулу (12) и интегрируя по формуле (12), получаем вероятность выжигания $P\left(\Omega_{01}\right)$ при двухступенчатом ФСП в виде следующих трех слагаемых $\left[{ }^{13}\right]$ :

$$
P\left(\Omega_{01}\right)=P_{22}\left(\Omega_{01}\right)=P_{2}\left(\Omega_{01}\right)+P_{2}\left(\Omega_{01}-\omega_{1}\right)+P_{2 i}\left(\Omega_{01}\right) .
$$

Вероятности $P_{2}\left(\Omega_{01}\right)$ и $P_{2}\left(\Omega_{01}-\omega_{1}\right)$ соответствуют выжиганию селектирующими импульсами соответственно на частотах $\omega_{0}$ и $\omega_{0}+\omega_{1}$ и определяются формулой (16) (для получения вероятности $P_{2}\left(\Omega_{01}-\omega_{1}\right)$ надо заменить в (16) $\omega_{0}$ на $\left.\omega_{0}+\omega_{1}\right)$. Вероятность $P_{2 i}\left(\Omega_{01}\right)$ соответствует выжиганию интерференционным членом и имеет следующий вид:

$$
\begin{aligned}
& P_{2 i}\left(\Omega_{01}\right)=\varepsilon \Delta \exp (-\Delta T)\left\{\left[\left(-2 \omega_{1} x+a(\Gamma+a)\right) \xi_{1}^{-1}+\right.\right. \\
& \left.+\left(2 \omega_{1}\left(x-\omega_{1}\right)+a(\Gamma+a)\right) \xi_{3}^{-1}\right] \xi_{5}^{-1} \cos \left(\omega_{1} \tau_{2}\right)+ \\
& \left.+\left[\left(2 a x+\omega_{1}(a+\Gamma)\right) \xi_{1}^{-1}+\left(-2 a\left(x-\omega_{1}\right)+\omega_{1}(a+\Gamma)\right) \xi_{3}^{-1}\right] \xi_{5}^{-1} \sin \left(\omega_{1} \tau_{2}\right)\right\}- \\
& -\varepsilon \Delta \exp \left(-\gamma_{1} T\right)\left\{\left[\left(2 \omega_{1} x+a(\Gamma-a)\right) \xi_{2}^{-1}+\left(-2 \omega_{1}\left(x-\omega_{1}\right)+\right.\right.\right. \\
& \left.+a(\Gamma-a)) \xi_{4}^{-1}\right] \xi_{5}^{-1} \cos \left(\omega_{1} \tau_{1}\right)+\left[\left(-2 a x+\omega_{1}(\Gamma-a)\right) \xi_{2}^{-1}+\right. \\
& \left.\left.+\left(2 a\left(x-\omega_{1}\right)+\omega_{1}(\Gamma-a)\right) \xi_{4}^{-1}\right] \xi_{5}^{-1} \sin \left(\omega_{1} \tau_{1}\right)\right\}- \\
& -\varepsilon \Delta \exp \left(-\frac{1}{2}\left(\Gamma+\gamma_{1}+\Delta\right) T\right)\left\{\left[2 \xi_{6} \cos y_{1}+\right.\right. \\
& \left.+\left(\omega_{1}(\Gamma+a)-2 \Gamma x\right) \sin y_{1}\right] \xi_{1}^{-1} \xi_{4}^{-1}+ \\
& \left.+\left[2 \xi_{6} \cos y_{2}+\left(-\omega_{1}(\Gamma-a)+2 \Gamma x\right) \sin y_{2}\right] \xi_{2}^{-1} \xi_{3}^{-1}\right\},
\end{aligned}
$$

где

$$
\begin{array}{ll}
\xi_{3}=\left(x-\omega_{1}\right)^{2}+\frac{1}{4}(\Gamma+a)^{2}, & \xi_{6}=\left(x-\omega_{1}\right) x+\frac{1}{4}\left(a^{2}-\Gamma^{2}\right), \\
\xi_{4}=\left(x-\omega_{1}\right)^{2}+\frac{1}{4}(\Gamma-a)^{2}, & y_{1}=x \tau_{1}-\left(x-\omega_{1}\right) \tau_{2}, \\
\xi_{5}=\omega_{1}^{2}+a^{2}, & y_{2}=x \tau_{2}-\left(x-\omega_{1}\right) \tau_{1} .
\end{array}
$$

По формулам (15) и (22) и с учетом формул (16) и (23) произведены расчеты, которые представлены на рис. 3 .

Штриховая линия показывает контур провала, получаемого при выжигании одновременно двумя селектирующими импульсами на час- 
тотах $\omega_{0}$ и $\omega_{0}+\omega_{1}$ в двухуровневой системе. В трехуровневой системе эти же два селектирующих импульса приводят к появлению двух провалов (сплошная линия) по мере увеличения задержки $T$. Таким образом, используя данное двухступенчатое выжигание, значительно увеличивается спектральное разрешение метода ФСП.

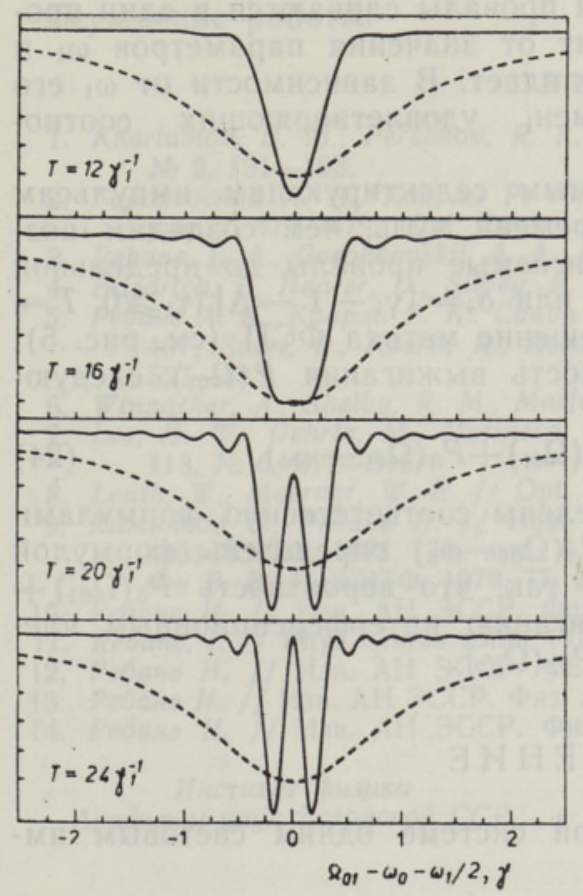

Рис. 3. Провалы в ФНР $\varrho\left(\Omega_{01}\right)$ при двухступенчатом (сплошная линия) и одноступенчатом (штриховая линия) ФСП двумя селектирующими импульсами. Параметры: $\Gamma=0, \Delta=0,99 \gamma_{1}, \omega_{1}=0,25 \gamma_{1} \quad\left(\sigma_{0}=\gamma_{1}, \sigma_{1}=\right.$ $\left.=1,99 \gamma_{1}, \quad \sigma_{2}=0,01 \gamma_{1}\right)$.

Рис. 5. Провалы в ФНР $\mathrm{Q}\left(\Omega_{01}\right)$ при одноступенчатом (штриховая линия) и двухступенчатом (штрих-пунктир) ФСП двумя селектирующими импульсами, а также при двухступенчатом ФСП суммой селектирующих импульсов и добавочного $\delta$-импульса (сплошная линия). Параметры: $\Gamma=4 \gamma_{1}, \quad \Delta=4,5 \gamma_{1}, \quad \tau_{2}=\gamma_{1}^{-1}, \quad \omega_{1}=\gamma_{1} \quad\left(\sigma_{0}=\right.$ $\left.=5 \gamma_{1}, \quad \sigma_{1}=9,5 \gamma_{1}, \quad \sigma_{2}=7,5 \gamma_{1}, \quad \sigma_{3}=0,5 \gamma_{1}\right)$.

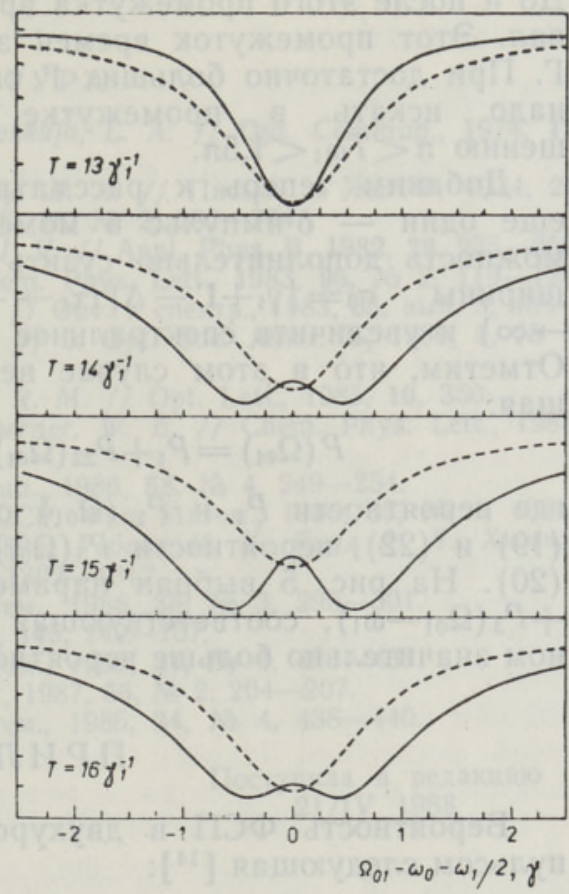

Рис. 4. То же, что и на рис. 3. Параметры: $\Gamma=0,5 \gamma_{1}, \Delta=0,01 \gamma_{1}, \omega_{1}=0,25 \gamma_{1} \quad\left(\sigma_{0}=\right.$ $\left.=1,5 \gamma_{1}, \quad \sigma_{1}=1,51 \gamma_{1}, \quad \sigma_{2}=1,49 \gamma_{1}\right)$.

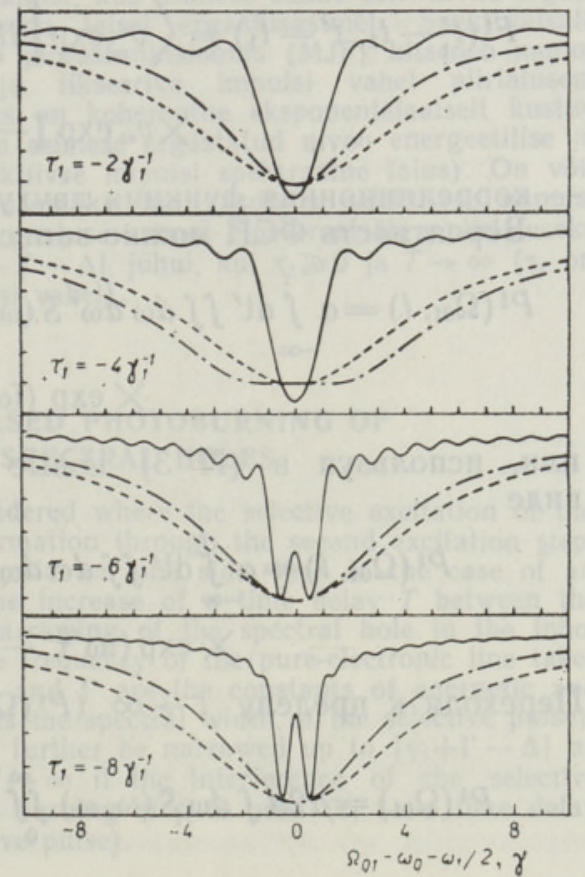


В заключение отметим еще одно обстоятельство. Расчеты по формулам (15) и (22) (с учетом (16) и (23)) при значениях $0 \simeq \Delta<\gamma_{1}$ показывают (см. рис. 4), что даже в случае, когда сдвиг центральных частот селектирующих импульсов $\omega_{1}$ значительно меньше ширины провала $\sigma$, выжигаемого каждым из этих импульсов, существует промежуток значений времен задержки $T$, при котором имеются два провала. До и после этого промежутка времени провалы сливаются в один провал. Этот промежуток времен зависит от значения параметров $\omega_{1}$ и Г. При достаточно больших Г он пропадает. В зависимости от $\omega_{1}$ его надо искать в промежутке времен, удовлетворяющих соотношению $\pi<T_{\omega_{1}}<1,5 \pi$.

Добавим теперь к рассматриваемым селектирующим импульсам еще один - $\delta$-импульс в момент времени нуль, чем создадим возможность дополнительно сузить выжигаемые провалы до предельной ширины $\quad \sigma_{3}=\left|\gamma_{1}+\Gamma-\Delta\right|\left(\tau_{1} \rightarrow-\infty\right)$ или $\sigma_{4}=\left|\gamma_{1}-\Gamma-\Delta\right|\left(\tau_{1} \geqslant 0, T \rightarrow\right.$ $\rightarrow \infty)$ и увеличить спектральное разрешение метода ФСП (см. рис. 5). Отметим, что в этом случае вероятность выжигания $P\left(\Omega_{01}\right)$ следующая:

$$
P\left(\Omega_{01}\right)=P_{1}+P_{22}\left(\Omega_{01}\right)+P_{3}\left(\Omega_{01}\right)+P_{3}\left(\Omega_{01}-\omega_{0}\right),
$$

где вероятности $P_{1}$ и $P_{22}\left(\Omega_{01}\right)$ определены соответственно формулами (19) и $(22)$, вероятности $P_{3}\left(\Omega_{01}\right)$ и $P_{3}\left(\Omega_{01}-\omega_{0}\right)$ определены формулой (20). На рис. 5 выбран параметр $d$ так, что вероятность $P_{3}\left(\Omega_{01}\right)+$ $+P_{3}\left(\Omega_{01}-\omega_{1}\right)$, соответствующая выжиганию интерференционным членом значительно больше вероятности $P_{22}\left(\Omega_{01}\right)$.

\section{П Р Л О ЖЕ Н И}

Вероятность ФСП в двухуровневой системе одним световым импульсом следующая $\left[{ }^{14}\right]$ :

$$
P^{1}\left(\Omega_{01}, t\right)=\alpha \int_{-\infty}^{t} \mathrm{dt}^{\prime} \int_{-\infty}^{t^{\prime}} \int_{-\infty} \mathrm{dt}_{1} \mathrm{dt}_{1}^{\prime} S\left(t_{1}, t_{1}^{\prime}\right) F^{4}\left(t_{1}^{\prime}-t_{1}, t^{\prime}-t_{1}\right),
$$

где

$$
\begin{aligned}
F^{1}\left(t_{1}^{\prime}-t_{1}, t^{\prime}-t_{1}\right)= & \left\langle v_{\omega}^{+} \exp \left[i \hat{H}\left(t_{1}^{\prime}-t_{1}\right)-\frac{1}{2} \hat{\gamma}\left(2 t^{\prime}-t_{1}-t_{1}^{\prime}\right)\right] \times\right. \\
& \left.\times v_{\omega} \exp \left[-i \hat{H}\left(t_{1}^{\prime}-t_{1}\right)\right]\right\rangle
\end{aligned}
$$

- корреляционная функция двухуровневой системы.

Вероятность ФСП можно записать также в виде

$$
\begin{gathered}
P^{1}\left(\Omega_{01}, t\right)=\alpha \int_{-\infty}^{t} \mathrm{dt}^{\prime} \iint d \omega d \omega^{\prime} S\left(\omega, \omega^{\prime}\right) \iint_{-\infty}^{t^{\prime}} \mathrm{dt}_{1} \mathrm{dt}_{1}^{\prime} F^{1}\left(t_{1}^{\prime}-t_{1}, t^{\prime}-t_{1}\right) \times \\
\times \exp \left(i \omega t_{1}-i \omega^{\prime} t_{1}^{\prime}\right)
\end{gathered}
$$

или, используя в (П 3) новые переменные $\tau=t^{\prime}-t_{1}, \tau^{\prime}=t^{\prime}-t_{1}{ }^{\prime}$, в. виде

$$
\begin{aligned}
P^{1}\left(\Omega_{01}, t\right)= & \alpha \int_{-\infty}^{t} \mathrm{dt}^{\prime} \iint d \omega d \omega^{\prime} S\left(\omega, \omega^{\prime}\right) \iint_{0}^{\infty} d \tau d \tau^{\prime} F^{1}\left(\tau, \tau^{\prime}\right) \times \\
& \times \exp \left(i \omega^{\prime} \tau^{\prime}-i \omega \tau+i\left(\omega-\omega^{\prime}\right) t^{\prime}\right) .
\end{aligned}
$$

Переходя к пределу $t \rightarrow \infty \quad\left(P^{1}\left(\Omega_{01}\right) \equiv \lim _{t \rightarrow \infty} P^{1}\left(\Omega_{01}, t\right)\right)$, получим

$$
P^{1}\left(\Omega_{01}\right)=\alpha 2 \pi \int d \omega \widetilde{S}(\omega, \omega) \iint_{0}^{\infty} d \tau d \tau^{\prime} F^{1}\left(\tau, \tau^{\prime}\right) \exp \left[i \omega\left(\tau^{\prime}-\tau\right)\right] .
$$


В случае стационарного ФСП в промежутке времени $(0, t)\left(t \gg \gamma^{-1}\right)$ вероятность выжигания следующая:

$$
P_{\text {ст }}^{1}\left(\Omega_{01}, t\right)=\alpha t \int d \omega S(\omega) \int_{0}^{\infty} d \tau d \tau^{\prime} F^{1}\left(\tau, \tau^{\prime}\right) \exp \left[i \omega\left(\tau^{\prime}-\tau\right)\right] .
$$

Автор признателен К. К. Ребане, В. Хижнякову и Я. Кикасу за обсуждение работы.

\section{ЛИТЕРАТ У РА}

1. Kharlamov, B. M., Personov, R. I., Bykovskaja, L. A. // Opt. Commun., 1974, 12, № 2, 191-193.

2. Гороховский А. А., Каарли Р. К., Ребане Л. А. // Письма в ЖЭТФ, 1974, 20, вып. 7, 474-479: Ont. Commun.. 1976, 16, № 2, 282-284.

3. Rebane, L. A., Gorokhovskii, A. A., Kikas, J. V. // Appl. Phys. B, 1982, 29, 235-250.

4. Friedrich, J., Haarer, D., Silbey, R. // Chem. Phys. Lett., 1983, 95, № 2, 119-123.

5. Ребане А. К., Каарли Р. К.. Саари П. М. // Опт. и спектр., 1983, 55, вып. 3, 405407; Saari, P., Kaarli, R., Rebane, A. // J. Opt. Soc. Amer. B, 1986, 3, № 4, $527-533$.

6. Winnacker, A., Shelby, R. M., Macfarlane, R. M. // Opt. Lett., 1985, 10, 350.

7. Lee, H. W., Gehrtz, M., Marinero, E., Moerner, W. E. // Chem. Phys. Lett., 1985, 118, № $6,611-616$.

8. Lenth, W., Moerner, W. E. // Opt. Commun., 1986, 58, № 4, 249-254.

9. Хижняков В., Ребане И. // Изв. АН ЭССР. Физ. Матем., 1986, 35, № 4, 406415; ЖЭТФ, 1978, 74, вып. 3, 885-896; Ребане И. К., Туул А. Л., Хижняков В. В. // ЖЭТФ, 1979, 77, вып. 4, 1302-1312.

10. Ребане И. // Изв. АН ЭССР. Физ. Матем., 1986, 35, № 3, 296-301.

11. Rebane, I. // Phys. status solidi (b), 1988, 145, 749-757.

12. Ребане И. // Изв. АН ЭССР. Физ. Матем., 1988, 37, № 4, 428-431.

13. Ребане И. // Изв. АН ЭССР. Физ. Матем., 1987, 36, № 2, $204-207$.

14. Ребане И. // Изв. АН ЭССР. Физ. Матем., 1985, 34, № 4, 438-440.
Ннститут физики
Академиш наук Эстонской ССР
Поступила в редакцию 21/IV 1988

\section{Inna REBANE \\ PIIRKITSASTE SPEKTRAALSÄLKUDE KAHEASTMELISE IMPULSSVALGUSPOLETAMISE TEOORIA}

On vaadeldud kaheastmelist spektraalsälkamist, kus esimese astme selektiivne ergastus «fikseeritakse» fotokeemilise muundumisega teisel ergastusastmel. Spektraalsälk puhtelektronjoone sageduse mittehomogeenses jaotusfunktsioonis (MJF) kitseneb monotoonselt viivise $T$ kasvades selektiivse ja fikseeriva impulsi vahel piirlaiuseni $\Gamma+\left|\gamma_{1}-\Delta\right|$ juhul, kui selektiivseks impulsiks on koherentne eksponentsiaalselt kustuv impulss ja fikseerivaks $\delta$-impulss ( $\gamma_{1}$ ja $\Gamma$ on esimese ergastatud nivoo energeetilise ja faasilise relaksatsiooni konstandid, $\Delta-$ selektiivse impulsi spektraalne laius). On vốimalik muuta spektraalsälku MJF-s veelgi kitsamaks, kui kasutada selektiivse impulsi interferentsi täiendava $\delta$-impulsiga esimesel ergastuse astmel. Spektraalsälgu piirlaiuseks on $\left|\gamma_{1}+\Gamma-\Delta\right|$ juhul, kui $\tau_{1} \rightarrow-\infty$, ja $\left|\gamma_{1}-\Gamma-\Delta\right|$ juhul, kui $\tau_{1} \geqslant 0$ ja $T \rightarrow \infty\left(\tau_{1}\right.$ on viivis täiendava $\delta$-impulsi ja selektiivse impulsi vahel).

\section{Inna REBANE}

\section{THEORY OF TWO-STEP PULSED PHOTOBURNING OF LIMITING NARROW SPECTRAL HOLES}

A two-step spectral hole burning is considered where the selective excitation of the first step is fixed by a photochemical transformation through the second excitation step. If the selective pulse is coherent and exponential on one side, then in the case of an extremely short fixing pulse ( $\delta$-pulse), on the increase of a time delay $T$ between the selective and fixing pulses, a monotonous narrowing of the spectral hole in the inhomogeneous distribution function (IDF) of the frequency of the pure-electronic line takes place up to the limit width $\Gamma+\left|\gamma_{1}-\Delta\right|\left(\gamma_{1}\right.$ and $\Gamma$ are the constants of energetic and phase relaxation of the first excited level, $\Delta$ is the spectral width of the selective pulse). It is shown that the spectral hole in IDF can further be narrowed up to $\left|\gamma_{1}+\Gamma-\Delta\right|$ at $\tau_{1} \rightarrow-\infty$, or $\left|\gamma_{1}-\Gamma-\Delta\right|$ at $\tau_{1} \geqslant 0$, and $T \rightarrow \infty$ if the interference of the selective pulse with an additional $\delta$-pulse at the first burning step is used $\left(\tau_{1}-\right.$ a time delay between the additional $\delta$-pulse and the selective pulse). 